\title{
A proposed model for the flowering signaling pathway of sugarcane under photoperiodic control
}

\author{
C.P. Coelho ${ }^{1}$, A.P. Costa Netto ${ }^{2}$, J. Colasanti ${ }^{3}$ and A. Chalfun-Junior ${ }^{1}$ \\ ${ }^{1}$ Departamento de Biologia, Setor de Fisiologia Vegetal, \\ Laboratório de Fisiologia Molecular de Plantas, \\ Universidade Federal de Lavras, Lavras, MG, Brasil \\ ${ }^{2}$ Curso de Ciências Biológicas, Universidade Federal de Goiás, Campus Jataí, \\ Jataí, GO, Brasil \\ ${ }^{3}$ Department of Molecular and Cellular Biology, University of Guelph, Guelph, \\ ON, Canada
}

Corresponding author: A. Chalfun-Junior

E-mail: chalfunjunior@dbi.ufla.br

Genet. Mol. Res. 12 (2): 1347-1359 (2013)

Received July 30, 2012

Accepted January 25, 2013

Published April 25, 2013

DOI http://dx.doi.org/10.4238/2013.April.25.6

ABSTRACT. Molecular analysis of floral induction in Arabidopsis
has identified several flowering time genes related to 4 response
networks defined by the autonomous, gibberellin, photoperiod, and
vernalization pathways. Although grass flowering processes include
ancestral functions shared by both mono- and dicots, they have
developed their own mechanisms to transmit floral induction signals.
Despite its high production capacity and its important role in biofuel
production, almost no information is available about the flowering
process in sugarcane. We searched the Sugarcane Expressed Sequence
Tags database to look for elements of the flowering signaling pathway
under photoperiodic control. Sequences showing significant similarity
to flowering time genes of other species were clustered, annotated, and
analyzed for conserved domains. Multiple alignments comparing the
sequences found in the sugarcane database and those from other species 
were performed and their phylogenetic relationship assessed using the MEGA 4.0 software. Electronic Northerns were run with Cluster and TreeView programs, allowing us to identify putative members of the photoperiod-controlled flowering pathway of sugarcane.

Key words: Floral induction; Photoperiodism; Saccharum spp; Flowering time genes; SUCEST; Biomass yield

\section{INTRODUCTION}

Transition from vegetative to reproductive growth is an important event in the development of higher plants. The change to a reproductive program is manifested in vegetative tissues and is regulated by both environmental and endogenous factors. The shoot apical meristem (SAM) is a population of undifferentiated cells that produce leaves and branches during vegetative growth. Under environmental and endogenous responses, SAM undergoes an identity change and produces floral primordia. Molecular analysis of floral induction has been extensively developed in Arabidopsis (Simpson and Dean, 2002). These studies have identified numerous flowering time genes that act in 4 response networks: autonomous, gibberellin (Baurle and Dean, 2006), photoperiod, and vernalization (Mouradov et al., 2002; Parcy, 2005).

The light-dependent flowering pathway can be controlled by 2 mechanisms; light quality and day length (Bernier and Periellux, 2005). These environmental factors lead to a cascade of responses that directly affect expression of the Phytochrome B, Cryptochrome 2, and Phytochrome A genes. A circadian clock-controlled mechanism integrates the inductive long-day (LD) photoperiod signals, which leads to the expression of Gigantea $(G I)$, followed by the activation of Constans $(C O)$ expression, and finally the induction of the Flowering locus $T(F T)$ gene. The $F T$ gene product acts as a leaf-synthesized florigen that migrates through the phloem to the SAM to cause flowering.

Although grass flowering processes utilize some functions also found in dicots, they also have developed their own mechanisms to produce floral induction signals (Colasanti and Coneva, 2009). Rice plants possess some genes that are absent in dicot plants, such as GHD7 (Grain number, Plant Height, and Heading Date7) and EHD1 (Early heading date1) (Greenup et al., 2009). These genes, which integrate information about short-day (SD)-induced flowering, act independently of the GI-CO-FT pathway.

Sugarcane, a monocot plant, is the main source of sugar production, representing almost two-thirds of the world production. A better understanding of the flowering process in sugarcane, an SD plant, is important because, among other factors, it is related to crop yield. The transition to reproductive growth leads to translocation of some of the sugar to the developing inflorescence, thus diverting the stored sugar away from stalks and, consequently, decreasing crop sugar yield.

Studies involving the flowering process in this crop plant will contribute to future insights into sugarcane genetics, physiological processes related to sucrose content/translocation, and the use of biotechnology approaches to increase sugar production. However, research related to the characterization of sugarcane flowering time genes is scarce.

The Sugarcane EST project (Figueiredo et al., 2001), SUCEST, encompasses a collection of 240,000 ESTs generated from 26 cDNA libraries constructed from different organs and/or tissues at different developmental stages, including apical meristem, flowers, leaf roll, 
seeds, internode, stem bark, etiolated leaves, and lateral buds (Vettore et al., 2001).

In this study, we employed in silico analyses to search the SUCEST database for putative orthologs of flowering time genes that are involved in the photoperiod-controlled floral inductive signaling pathway in sugarcane.

\section{MATERIAL AND METHODS}

\section{Database searches and alignments}

Homologs of functionally characterized genes involved in the flowering signaling pathway under photoperiodic control $(G I \rightarrow C O \rightarrow F T)$ were identified by data mining in the SUCEST database (http://compbio.dfci.harvard.edu/tgi/plant.html) using plant gene (BLASTn) and protein (tBLASTn) sequences as bait. Sequences with significant similarity (e-value $>10^{-4}$ ) were selected and submitted to clustering by the CAP3 program (Huang and Madan, 1999), forming the EST contigs and singlets.

The Saccharum officinarum EST-contigs and EST-singlets obtained were manually annotated, and data validation was performed by local tBLASTx and tBLASTn searches of the retrieved sequences against the GenBank database. Selected sequences were then used as bait in another search against the SUCEST database, aiming at uncovering additional reads, as well as to remount incomplete clusters. This process was repeated until no more new significant reads were found. Open reading frames (ORFs) of validated sequences were obtained through the ORFinder tool, from NCBI (http://www.ncbi.nlm.nih.gov), and their protein sequences were generated through the translation tool found in the ExPASY (http://www.expasy.ch) protein database. The protein sequence alignments were performed by the ClustalW program (Thompson et al., 1994), using default parameters.

\section{Phylogenetic analysis}

The putative orthology of the deduced amino acid sequences of sugarcane transcripts, compared to homologs from other species, was assessed by phylogenetic trees formed by the MEGA software, version 4.0 (Tamura et al., 2007), with the neighbor-joining comparison model (Saitou and Nei, 1987), $p$-distance method and pairwise suppression. Bootstrap values from 1000 replicates were used to assess the robustness of the trees.

\section{In silico gene expression analysis}

In silico qualitative gene expression profiling was performed using virtual Northern blot analyses. For each EST-contig and EST-singlet, frequencies of reads that form each ESTcontig and EST-singlet in the libraries in which they were expressed were calculated. This procedure required that the data have been previously normalized to give a more accurate idea of the degree of expression of the sequences in each treatment and plant organ when all libraries were considered in this study.

Normalization consisted of multiplying each read by the quotient between the number of reads from the library where it was expressed and the sum of reads of all libraries where expression was found. The results were plotted in a matrix and gene expression patterns among 
ESTs and libraries were obtained by hierarchical clustering, performed by the Cluster v.3 program (Eisen et al., 1999). Graphic outputs were generated by the TreeView v.1.6 software (Eisen et al., 1999) and presented in a color scale from black to red, where red indicated higher expression levels. Undetectable expression was noted in gray.

\section{RESULTS}

The main components of the flowering pathway under photoperiodic control were compiled, and their sequences were used to search sugarcane EST-contigs. Taken together, the results of the phylogenetic analyses, electronic Northern and BLASTp searches allowed for the identification of candidates for several genes that may be involved in the sugarcane flowering pathway under photoperiodic control.

Analyses at the SUCEST database revealed 16 reads related to the GI gene, clustered into 7 contigs and 2 singlets. As shown in the phylogenetic tree (Figure 1A) and in Table 1, $\mathrm{GiC} 2$ and GiC5 peptide candidates showed high similarity with maize $G I(\mathrm{ZmGI})$, with amino acid identity ranging from 94 to $97 \%$. It was possible to divide the phylogenetic tree into 2 subgroups, with one group corresponding to neutral day plants, which included $\mathrm{GiC} 2$ and another group corresponding to LD and SD plants, including the GiC5. The electronic Northern showed that these contigs are expressed in 6 (GiC2) and 12 (GiC5) different libraries, in no tissue-specific manner, including tissues where the GI typically acts, such as apical meristem surrounding immature and mature leaves and in the inflorescence. BLASTp analyses revealed high identity of $\mathrm{GiC} 2(94 \%)$ and $\mathrm{GiC} 5(97 \%)$ with a $\mathrm{Zm} \mathrm{GI}$ ortholog.

Twenty-three reads related to $C O$ were found and clustered into 1 contig and 8 singlets. The motif analyses showed that all sequences contained conserved domains for the family of $C O$-like genes, and they could be categorized into the 3 subgroups of $C O$ family genes (Griffiths et al., 2003; Wenkel et al., 2006). The results for $C O$ gene similarity (Figure 1B) indicated that sugarcane possesses some candidates for each subtype of the $C O$-like superfamily. This result was confirmed by BLASTp analyses, since the candidates found could be related to COL1, COL5, COL6, and COL10. Additionally, contig CoS1 was considered a candidate for the $C O$ gene because it showed a high level of similarity with the maize HD1 protein (81\% identity). Phylogenetic analyses showed that this singlet was grouped with $C O$ orthologs of related species, such as rice and maize, and the electronic Northern suggested that its expression was specific to inflorescence tissue.

Candidates for putative EHD1 and GHD7 orthologs were clustered into 5 contigs and 4 singlets derived from 20 reads, and 4 contigs and 2 singlets derived from 11 reads, respectively. It was possible to identify some candidates for EHDI and GHD7, which are monocotspecific genes, in the SUCEST database. According to phylogenetic analyses and the electronic Northern, Ehd1C4 is closely related to the rice EHD1 gene (OsEHD1) and is detected in leaf libraries, such as mature leaf tissues (Figure 1C). After comparing conserved domains, it was possible to identify the CCT domain of the GHD7 gene. The candidate Ghd7C4 (Figure 1D) showed very high abundance in the mature leaf tissue library. Through BLASTp analyses, Ghd7C4 was found to correlate with the barley COL7 gene, with an identity of $67 \%$.

It was possible to detect 20 reads for the $F T$ gene in searches, clustered into 3 contigs and 5 singlets, all of them containing the PEBP conserved domain and with high similarity to the maize ZCN superfamily (Danilevskaya et al., 2008). BLASTp results revealed 2 can- 
didates for Terminal Flower1 (TFL1)-like genes in the SUCEST database: FtC3 and FtS2. These candidates showed high similarity to a rice putative $T L F 1$ ( 83 and $79 \%$, respectively), and an electronic Northern showed that these sequences are expressed in the leaves (FtS2) and lateral bud (FtC3) tissues. Results of the phylogenetic analyses suggested that the FtS1 and FtS5 EST-contigs were the most related to ZCN26 (Figure 1E), and the electronic Northern showed expression in root-shoot transition libraries. Phylogenetic results suggested that $\mathrm{FtC} 2$ and $\mathrm{FtC} 1$ are candidates of the $F T$-like I group orthologs, ZCN14-ZCN15 and ZCN19-ZCN25, respectively. FtS2, $\mathrm{FtC} 3, \mathrm{FtS1}$, and $\mathrm{FtS} 5$ could be categorized as candidates for the $F T$-like II group, related to ZCN8 and ZCN26. A candidate for a Mother of FT (MFT)-like subfamily gene, ZCN11, was also found, i.e., the FtS4 EST-contig.

A

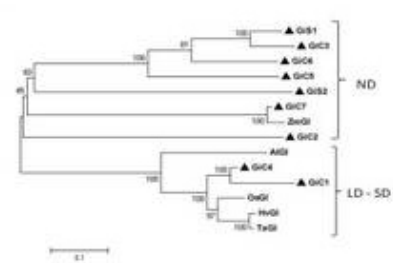

B
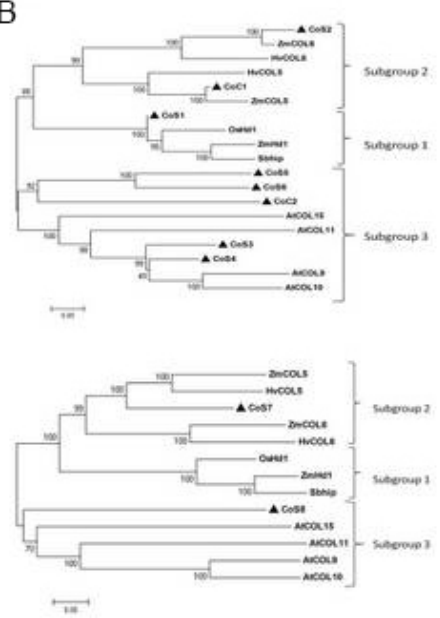

C

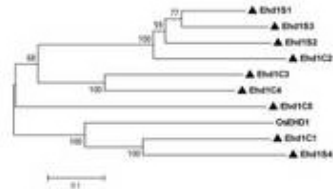

$\mathrm{D}$

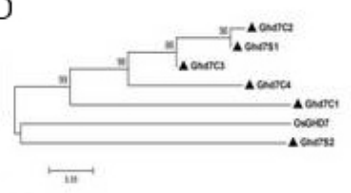

$\mathrm{E}$

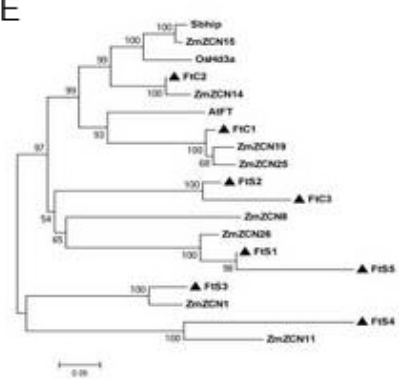

Figure 1. Phylogenetic analyses involving the sugarcane putative genes of the flowering pathway under photoperiodic control (triangles) and homolog sequences obtained from the NCBI database related to A. GI; B. $C O$; C. $E H D 1 ;$ D. GDH7; E. FT. Neighbor-joining trees were built for sugarcane-deduced amino acids and protein sequences from other species aligned with ClustalW2. Bootstrap values from 1000 replications were used to assess the robustness of the trees. Genetic distances are shown at the given scales. The protein sequences from other species and their respective accession numbers are as follows: A. Zea mays [ZmGI (ABZ81992.1)], Arabidopsis thaliana [AtGI (AAF00092.1)], Oryza sativa [OsGI (BAD68052.1)], Hordeum vulgare [HvGI (ACM49849.1)], Triticum aestivum [TaGI (AAL08497.2)]. B. Arabidopsis thaliana [AtCOL9 (NP_187422.1), AtCOL10 (NP_199636.1), AtCOL11 (NP_193260.2), AtCOL15 (NP_174126.1)], Zea mays [ZmCOL5 (NP_001147679.1), ZmCOL6 (NP _001148229.1), ZmHd1 (ABW82153.1)], Hordeum vulgare [HvCOL5 (AAL99264.1), HvCOL6 (AAL99267.1)], Oryza sativa [OsHd1 (BAI59731.1)], Sorghum bicolor [Sbhip (XP 002436860.1)]. C. Oryza sativa [OsEHD1 (ABF95340.1)]. D. Oryza sativa [OsGHD7 (ACA14489.1)]. E. Zea mays [ZmZCN1 (ABW96224.1), ZmZCN8 (ABX11010.1), ZmZCN11 (NP 001106264.1), ZmZCN14 (NP 001106251.1), ZmZCN15 (ABW96237.1), ZmZCN19(NP_001106256.1), ZmZZCN25 (NP_001106257.1), ZmZCN26 (ABW96244.1)], Oryza sativa [OsHd3a (BAF15064.1)], Arabidopsis thaliana [AtFT (ĀAF03936.1)], Sorghum bicolor [Sbhip (XP_002436509.1)]. 


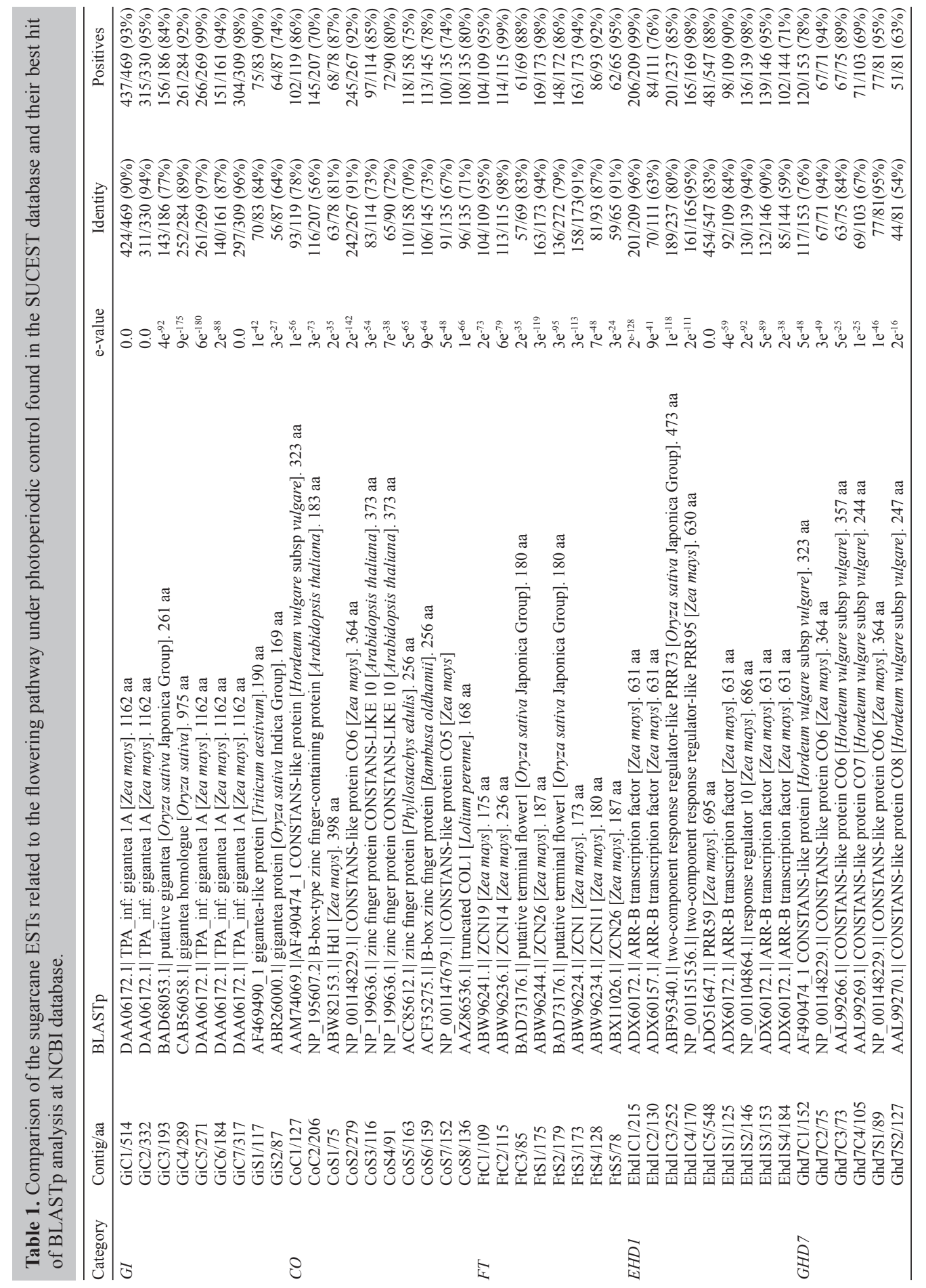




\section{DISCUSSION}

Previous studies in Arabidopsis and rice have shown that, although some pathways are conserved between mono- and dicot plants, each group has developed specific mechanisms to control the flowering process.

Almost nothing is known about the flowering pathway in sugarcane, although this process is extremely important and related to crop yield. A search for 5 flowering time genes in sugarcane, GI, CO, EHDI, GHD7, and FT, which are both mono- and dicot-specific genes, was performed in the SUCEST database.

\section{GI}

GI is a large protein that is nuclear localized (Huq et al., 2000) and regulates flowering through the integration of circadian rhythm periods, acting upstream of the $C O$ gene (Samach and Coupland, 2000). Analysis of this gene was performed since sugarcane floral induction is controlled by photoperiod and the circadian clock. The circadian clock is a pacemaker that controls rhythmic processes that occur within a period of $24 \mathrm{~h}$ (Hayama and Coupland, 2003). Mutation of GI impairs circadian rhythms and delays the flowering process, suggesting that its functions is to couple the circadian clock to the day-length perception inducing downstream genes, including those specifically related to the floral transition, such as $C O$ and FT (Mizoguchi et al., 2005). GI is highly conserved in higher plants, including monocot species such as rice (Hayama et al., 2002). A functional hierarchy of GI-CO-FT acting together to connect the circadian oscillator to the flowering pathway has been established (Mizoguchi et al., 2005). The 2 candidates found in sugarcane contain a conserved domain present in $G I$, although the sequences found encode incomplete ORFs. The contig GiC2 was grouped with GI proteins of day-neutral plants, and the GiC5 contig was grouped with GI of LD and SD plants. This information supports the idea that each candidate may act differently, depending on the environmental conditions in which sugarcane is subjected. Results from Higuchi et al. (2011) suggest that in Pharbitis nil, an SD plant with an absolute requirement for SD photoperiods to induce flowering, a $G I$ ortholog functions as a suppressor of flowering through the repression on an FT ortholog. Although sugarcane is an SD plant (Araldi et al., 2010), other variables interact with photoperiod signals to determine floral induction, such as low temperatures. Taken together with the electronic Northern (Figure 2A), it is possible to predict that the GI ortholog (GiC5) is important in the sugarcane flowering network, although its specific function (such as inducing or repressing downstream FT-like genes) needs to be confirmed by functional analyses.

\section{CO}

The $C O$ gene, downstream of $G I$, is a key regulatory protein that integrates signals from the circadian clock to control flowering (Putterill, 2001; Valverde, 2011). CO expression exhibits a circadian rhythm under continuous light, in which $C O$ has a diurnal expression pattern with a peak in the night, regulated by the circadian clock (Hayama and Coupland, 2003). Rice, which is an SD plant, has a $\mathrm{CO}$ ortholog (the $\mathrm{Hdl}$ gene) whose expression is repressed under LD and induced under SD floral inductive photoperiods (Izawa et al., 2003). Since sug- 
arcane is also an SD plant, a mechanism of flowering control via $C O$ orthologous genes may be shared between these 2 species. All the $\mathrm{CO}$-like candidates found contained an amino terminus B-box superfamily conserved domain, which regulates protein-protein interactions (Torok and Etkin, 2001), and/or the carboxyl terminus CCT domain. They could be grouped into specific classes of the $\mathrm{CO}$-like gene family. Cereals possess specific classes that are absent in Arabidopsis; i.e., the group I class genes that contain a single-B-box domain and the group IV class, which lacks the B-box domain and has only the CCT domain (Griffiths et al., 2003). In group IV, candidate genes were found in sugarcane. Complete ORFs of possible $C O$ orthologs in sugarcane could be found, such as $\mathrm{CoS} 1$, which has the B-box superfamily-conserved domain. $\mathrm{CoC} 1$ and $\mathrm{CoC} 2$ transcripts were present in different leaf libraries (Figure 2B). BLASTp analyses identified barley and Arabidopsis $\mathrm{CO}$-like genes; phylogenetic analyses suggested that they are related to subgroups I and III, respectively. The BLASTp and the phylogenetic results showed a closer relationship of $\mathrm{CoC} 1$ to monocot $C O$ orthologs, suggesting that this contig may be the candidate of $C O$ in sugarcane. Functional characterization needs to be performed to verify under which conditions (SD or LD) this gene is induced and/or repressed to determine whether or not this mechanism of control is shared between sugarcane and rice.

\section{EHD1}

A putative rice $E H D 1$ B-type response regulator (RR) domain was detected in all sugarcane EHD1 candidates found at the SUCEST database. In rice, this gene acts as a floral inducer under SD conditions by controlling the expression of the FT gene, and independently of the $H d l$ gene, induces expression of $F T$-like genes after SD treatment in $H d l$-deficient strains. Rice $H d 1$ is expressed only under SD conditions, but EHDl is expressed in both conditions, independent of $H d l$ (Doi et al., 2004). As in rice, a candidate sugarcane ortholog of EHD1 may perform this function. A complete ORF of Ehd1C4 was found to possess the RR-conserved domain. Additionally, this contig was detected in leaf libraries (Figure 2C), indicating that sugarcane may possess a 2-component flower signaling pathway, such as in rice plants (Doi et al., 2004; Endo-Higashi and Isawa, 2011).

\section{GHD7}

The GHD7 gene is responsible for inactivation of $E H D 1$ under LD conditions in rice. Similar to $E H D 1$, the $G H D 7$ gene is a monocot-specific gene, so far found only in rice, whose expression is related to crop grain number (Xue et al., 2008). The GHD7 protein contains a CCT motif, which mediates protein-protein interaction and nuclear localization, as found in $\mathrm{CO}$ proteins. GHD7 candidates found in sugarcane EST database are very similar to rice GHD7 and $\mathrm{CO}$-like genes because they share the same conserved domain. Due to this feature, BLASTp analyses found sequences related to $C O$ genes (Table 1). However, when the analyses for $C O$ genes were performed, these genes were not found, suggesting that they are GHD7 candidates. GHD7 contributes to the adaptation of rice cultivars to cold-climate regions (Xue et al., 2008), and it has been recently revealed that GHD7 transcription is mediated through phytochrome signaling and is gated in a photoperiod-dependent manner (Itoh et al., 2010). Electronic Northern results showed high abundance of potential GHD7 orthologs in sugarcane leaf tissue libraries, such as Ghd7C4, as in rice, where GHD7 is strongly expressed in 
the blades of fully expanded leaves (Xue et al., 2008). This suggests that this candidate may have an important function in the flowering process (Figure 2D), although expression in leaves alone does not mean that this gene is involved in the floral induction. There is a correlation between the EHD1 levels and GHD7 induction under non-inductive LD conditions, where GHD7 represses transcription of EHD1, thus subsequently affecting expression of Heading date $3 a$ (Hd3a, a rice florigen) (Itoh et al., 2010). The existence of this pathway may be predicted in sugarcane, since candidate orthologs were found in SUCEST.

\section{FT}

The FT gene, which encodes a phosphatidylethanolamine-binding protein (PEBP)related protein, is highly conserved in plants (Kobayashi et al., 1999). The FT protein has been found to move to the SAM, via the phloem, where it acts as a floral stimulus by activating the FD transcription factor (Corbesier et al, 2007). FT-related genes have been found in monocot plants such as wheat (19 genes), maize (30 genes, according to Chardon and Damerval, 2005; and 25 genes according to Danilevskaya et al., 2008), and rice (19 genes). Within the ZCN superfamily there are 3 major subfamilies: FT-like, $M F T$-like, and TFL1-like (Danilevskaya et al., 2008). Candidates for all members of the $Z C N$ superfamily could be found, including members of the $F T$-like I group, comprising monocot floral activators such as $H d 3 a$ (Danilevskaya et al., 2008). Maize possesses TFL1-like genes, named ZCN1 to ZCN6 genes, which when expressed ectopically modify the flowering time and inflorescence architecture in transgenic maize plants and maintain the indeterminacy of vegetative meristems (Danilevskaya et al., 2010). TLF1, which is a meristem identity gene, is an antagonist of the FT gene, despite the similarity in their sequences (Kobayashi et al., 1999; Tahery et al., 2009). The candidate FtS2 is a possible sugarcane $F T$ ortholog; it contains the complete PEBP superfamily domain, and transcripts have been found in mature leaf libraries (Figure 2E), as would be expected of a potential florigen-encoding gene. Despite a BLASTp analysis suggesting that $\mathrm{FtS} 2$ is highly similar to the TFL1 protein, which is an antagonist of the FT protein, FtS2 is expressed in mature leaf tissues, indicating that it is more likely an FT ortholog rather than TFL1, which is expressed in inflorescence meristem tissues (Tahery et al., 2009).

\section{ZCN8}

Recent findings show that $Z C N 8$ is an $F T$ ortholog gene in maize and teosinte (Lazakis et al., 2011; Meng et al., 2011). Exposure of teosinte plants to SD photoperiods that cause flowering is correlated with a large increase in $Z C N 8$ expression. A more moderate increase in $Z C N 8$ expression has been observed in maize, a neutral-day plant, under SD conditions. Other evidence has shown that ZCN8 is involved in photoperiod sensitivity, acting as a florigen, since ectopic expression in Arabidopsis rescues the FT mutant phenotype (Lazakis et al., 2011). Moreover, ZCN8-silencing experiments showed late flowering of transgenic maize plants (Meng et al., 2011). No clear ortholog of $Z C N 8 / F T$ was found in BLASTp searches, and the expression profile showed no transcript in leaf tissues (Figure 2E). However, through phylogenetic analyses, the FtS1 and FtS5 EST-contigs were the most related to ZCN8. Together, this information suggests that $Z C N$-like superfamily gene function is conserved between the species, and that some of them may act as floral activators in sugarcane. 
A

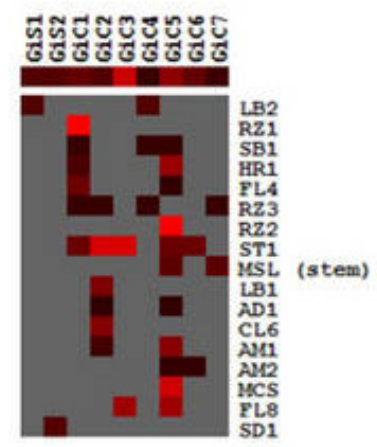

D

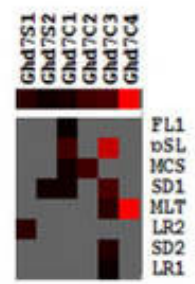

B
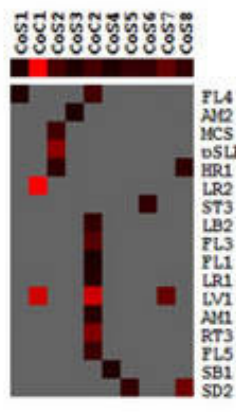

C

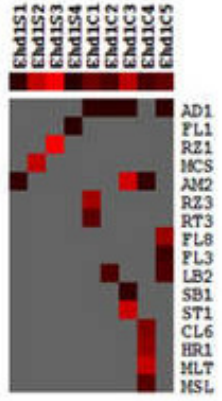

E

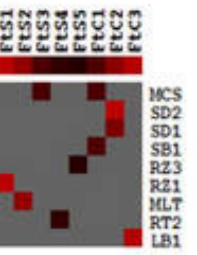

Figure 2. In silico expression profile of putative elements of the flowering pathway under photoperiodic control: A. Gigantea; B. Constans; C. Early heading date1; D. Heading date7; E. Flowering locus T. The normalized numbers of reads for the transcripts in each library are represented in a scale from black to red. The contigs (C) and singlets (S) are represented as columns and the sugarcane libraries as lines. Sugarcane libraries are as follows: FL4 (developed inflorescence and rachis), AM2 (apical meristem and tissues surrounding of immature plants), MCS (stem), pSRL (leaf roll including apex after floral induction), HR1 (seedling inoculated with Herbaspirilum rubrisubal), LR2 [(leaf roll from field-grown adult plants (small insert)], ST3 (fourth apical stalk internodes of adult plants), LB2 (lateral buds from adult plants), FL3 (base of developing inflorescence), FL1 (inflorescence at the beginning of development), LR1 [leaf roll from field-grown adult plants (large insert)], LV1 (etiolated leaves from in vitro-grown seedlings), AM1 (apical meristem and tissues surrounding of mature plants), RT3 (root apex from adult plants), FL5 (developed inflorescence), SB1 (stalk bark from adult plants), SD2 (developing seeds), AD1 (seedlings inoculated with Gluconacetobacter diazot), FL1 [inflorescence at beginning of development (1 $\mathrm{cm}$ long)], RZ1 [shoot-root transition zone from young plants (large insert)], RZ3 (shoot-root transition zone from adult plants), FL8 [developing inflorescence and rachis $(10 \mathrm{~cm}$ long)], ST1 (first apical stalk internodes of adult plants), CL6 [pool of sugarcane calli submitted to low temperature $\left(4^{\circ} \mathrm{C}\right)$ ], MLT (mature leaf tissue), MSL (sugarcane mature stem library), SD1 [developing seeds (large insert library)], RT2 [root tips (0.3 cm long) from adult plants], LB1 (lateral buds from field-grown adult plants), pSL (leaves after floral induction), RZ2 [shoot-root transition zone from young plants (small insert)].

A model for the photoperiodic mechanism of the flowering pathway (Figure 3) is proposed based on a comparative transcriptome and sequence conservation analysis. This preliminary study of the flowering time genes in sugarcane provides basic information for in-depth studies relating to the flowering process in this important crop. Further analyses of the genes identified will also provide a better understanding of the photoperiodic control of this crucial metabolic process. Moreover, functional characterization will help to unravel the molecular basis of the flowering process in different varieties of sugarcane with distinct florigenic potential. 


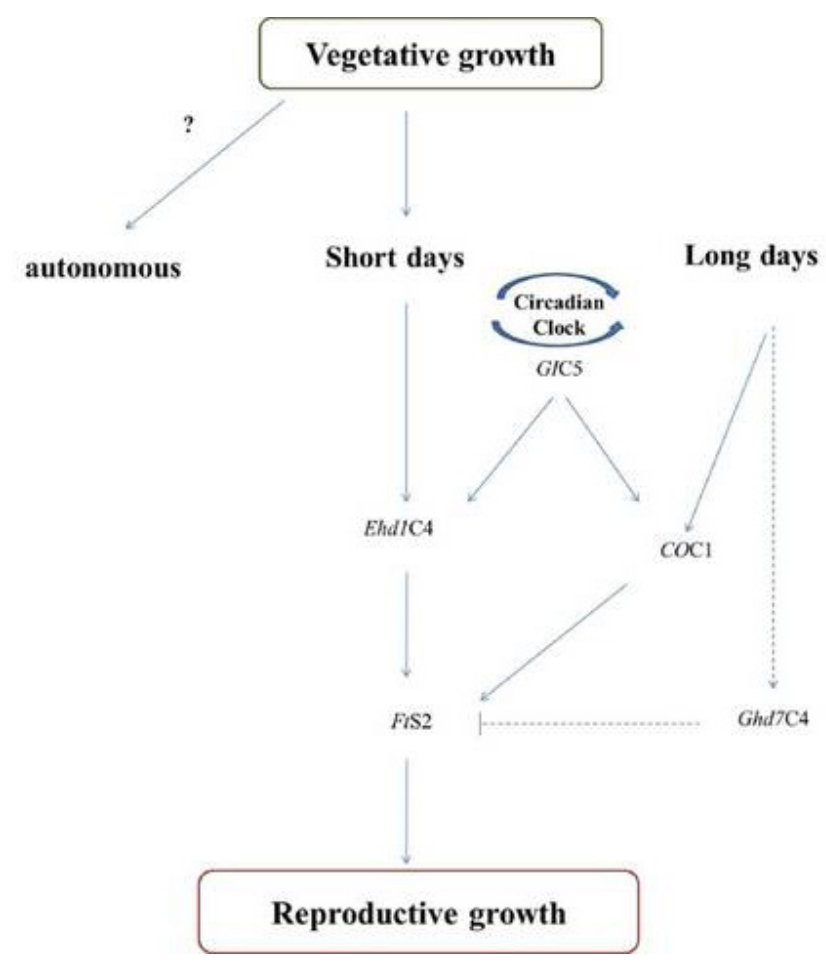

Figure 3. A hypothetical model of the gene network controlling floral induction under photoperiodic control in sugarcane. Photoperiodism and circadian clock controls the pathway by regulation of GI, EHD1, and CO ortholog genes. Two distinct mechanisms are assumed to be involved in the regulation of the $F T$ ortholog gene, one under long-day and short-day conditions, which induces FtS2 expression and another mechanism through the component GHD7 ortholog, which suppresses FtS2 expression in non-inducing conditions. Each element of the pathway corresponds to the EST-contig selected through the in silico characterization of the putative flowering time genes.

\section{ACKNOWLEDGMENTS}

Research supported by Conselho Nacional de Desenvolvimento Científico e Tecnológico $(\mathrm{CNPq})$, including fellowships and the "Fundação de Amparo à Pesquisa do Estado de Minas Gerais - FAPEMIG", for the financial support.

\section{REFERENCES}

Baurle I and Dean C (2006). The timing of developmental transitions in plants. Cell 125: 655-664.

Bernier G and Perilleux C (2005). A physiological overview of the genetics of flowering time control. Plant Biotechnol. J. 3: 3-16.

Chardon F and Damerval C (2005). Phylogenomic analysis of the PEBP gene family in cereals. J. Mol. Evol. 61: 579-590.

Colasanti J and Coneva V (2009). Mechanisms of floral induction in grasses: something borrowed, something new. Plant Physiol. 149: 56-62.

Corbesier L, Vincent C, Jang S, Fornara F, et al. (2007). FT protein movement contributes to long-distance signaling in floral induction of Arabidopsis. Science 316: 1030-1033.

Danilevskaya ON, Meng X, Hou Z, Ananiev EV, et al. (2008). A genomic and expression compendium of the expanded PEBP gene family from maize. Plant Physiol. 146: 250-264. 
Danilevskaya ON, Meng X and Ananiev EV (2010). Concerted modification of flowering time and inflorescence architecture by ectopic expression of TFL1-like genes in maize. Plant Physiol. 153: 238-251.

Doi K, Izawa T, Fuse T, Yamanouchi U, et al. (2004). Ehdl, a B-type response regulator in rice, confers short-day promotion of flowering and controls FT-like gene expression independently of Hd1. Genes Dev. 18: 926-936.

Eisen MB, Spellman PT, Brown PO and Botstein D (1999). Cluster analysis and display of genome-wide expression patterns. Proc. Natl. Acad. Sci. U. S. A. 95: 14863-14868.

Endo-Higashi N and Isawa T (2011). Flowering time genes Heading date 1 and Early heading date 1 together control panicle development in rice. Plant Cell Physiol. 52: 1083-1094.

Figueiredo RC, Brito MS, Figueiredo LHM, Quiapin AC, et al. (2001). Dissecting the sugarcane expressed sequence tag (SUCEST) database: unraveling flower-specific genes. Genet. Mol. Biol. 24: 77-84.

Greenup A, Peacock WJ, Dennis ES and Trevaskis B (2009). The molecular biology of seasonal flowering-responses in Arabidopsis and the cereals. Ann. Bot. 103: 1165-1172.

Griffiths S, Dunford RP, Coupland G and Laurie DA (2003). The evolution of CONSTANS-like gene families in barley, rice, and Arabidopsis. Plant Physiol. 131: 1855-1867.

Hayama R and Coupland G (2003). Shedding light on the circadian clock and the photoperiodic control of flowering. Curr. Opin. Plant Biol. 6: 13-19.

Hayama R, Izawa T and Shimamoto K (2002). Isolation of rice genes possibly involved in the photoperiodic control of flowering by a fluorescent differential display method. Plant Cell Physiol. 43: 494-504.

Higuchi Y, Sage-Ono K, Sasaki R, Ohtsuki N, et al. (2011). Constitutive expression of the GIGANTEA ortholog affects circadian rhythms and suppresses one-shot induction of flowering in Pharbitis nil, a typical short-day plant. Plant Cell Physiol. 52: 638-650.

Huang X and Madan A (1999). CAP3: A DNA sequence assembly program. Genome Res. 9: 868-877.

Huq E, Tepperman JM and Quail PH (2000). GIGANTEA is a nuclear protein involved in phytochrome signaling in Arabidopsis. Proc. Natl. Acad. Sci. U. S. A. 97: 9789-9794.

Itoh H, Nonoue Y, Yano M and Izawa T (2010). A pair of floral regulators sets critical day length for Hd3a florigen expression in rice. Nat. Genet. 42: 635-638.

Izawa T, Takahashi Y and Yano M (2003). Comparative biology comes into bloom: genomic and genetic comparison of flowering pathways in rice and Arabidopsis. Curr. Opin. Plant Biol. 6: 113-120.

Kobayashi Y, Kaya H, Goto K, Iwabuchi M, et al. (1999). A pair of related genes with antagonistic roles in mediating flowering signals. Science 286: 1960-1962.

Lazakis CM, Coneva V and Colasanti J (2011). ZCN8 encodes a potential orthologue of Arabidopsis FT florigen that integrates both endogenous and photoperiod flowering signals in maize. J. Exp. Bot. 62: 4833-4842.

Meng X, Muszynski MG and Danilevskaya ON (2011). The FT-like ZCN8 gene functions as a floral activator and is involved in photoperiod sensitivity in maize. Plant Cell 23: 942-960.

Mizoguchi T, Wright L, Fujiwara S, Cremer F, et al. (2005). Distinct roles of GIGANTEA in promoting flowering and regulating circadian rhythms in Arabidopsis. Plant Cell 17: 2255-2270.

Mouradov A, Cremer F and Coupland G (2002). Control of flowering time: interacting pathways as a basis for diversity. Plant Cell 14 (Suppl): S111-S130.

Parcy F (2005). Flowering: a time for integration. Int. J. Dev. Biol. 49: 585-593.

Putterill J (2001). Flowering in time: genes controlling photoperiodic flowering in Arabidopsis. Philos. Trans. R. Soc. Lond. B Biol. Sci. 356: 1761-1767.

Saitou N and Nei M (1987). The neighbor-joining method: a new method for reconstructing phylogenetic trees. Mol. Biol. Evol. 4: 406-425.

Samach A and Coupland G (2000). Time measurement and the control of flowering in plants. Bioessays 22: 38-47.

Simpson GG and Dean C (2002). Arabidopsis, the Rosetta stone of flowering time? Science 296: 285-289.

Tahery H, Abdullah MP, Norlia B, Kafilzadeh F, et al. (2009). Terminal flower 1 (TFL1) homolog genes in monocots. Eur. J. Sci. Res. 38: 26-37.

Tamura K, Dudley J, Nei M and Kumar S (2007). MEGA4: Molecular Evolutionary Genetics Analysis (MEGA) software version 4.0. Mol. Biol. Evol. 24: 1596-1599.

Thompson JD, Higgins DG and Gibson TJ (1994). CLUSTAL W: improving the sensitivity of progressive multiple sequence alignment through sequence weighting, position-specific gap penalties and weight matrix choice. Nucleic Acids Res. 22: 4673-4680.

Torok M and Etkin LD (2001). Two B or not two B? Overview of the rapidly expanding B-box family of proteins. Differentiation 67: 63-71.

Valverde F (2011). CONSTANS and the evolutionary origin of photoperiodic timing of flowering. J. Exp. Bot. 62: 2453-2463. 
Vettore AL, Silva FR da, Kemper EL and Arruda P (2001). The libraries that made SUCEST. Genet. Mol. Biol. 24: 1-7. Wenkel S, Turck F, Singer K, Gissot L, et al. (2006). CONSTANS and the CCAAT box binding complex share a functionally important domain and interact to regulate flowering of Arabidopsis. Plant Cell 18: 2971-2984.

Xue W, Xing Y, Weng X, Zhao Y, et al. (2008). Natural variation in Ghd7 is an important regulator of heading date and yield potential in rice. Nat. Genet. 40: 761-767. 